\title{
Perceived oral health in the UK and Australia
}

Impacts of oral disorders in the United Kingdom and Australia

\section{G. D. Slade, N. Nuttall, A. Sanders, J. Steele, F. Allen and S. Lahti Br Dent J 2005; 198: 489-493}

\section{Background}

Surveys of oral health have not previously compared national adult populations using measures of subjective oral health.

\section{Aims}

To compare subjective oral health of adults in the UK and Australian populations.

\section{Methods}

Cross sectional studies were conducted of people aged 18+ years in the 1998 UK Adult Dental Health Survey and the 1999 Australian National Dental Telephone Interview Survey. Subjective oral health was measured using the 14-item Oral Health Impact Profile questionnaire (OHIP-14).

Results

Among dentate people, the percentage reporting impacts 'fairly often' or 'very often' was marginally greater in Australia (18.2\%, $95 \%$ confidence interval $(\mathrm{CI})=16.2-20.2)$ than the UK $(15.9 \%$, $95 \% \mathrm{CI}=14.4-17.4)$. There were larger regional variations in prevalence within populations, ranging from $14.8 \%$ to $22.3 \%$ among Australian states/ territories, and from 13.6\% to $19.8 \%$ among countries within the UK. However, the mean number of impacts and rated severity of impacts was significantly greater in Australia than the UK

\section{Conclusions}

While the percentage of adults reporting adverse impacts of oral health was similar, Australians reported a larger number of impacts and more severe impacts than dentate people in the UK. Differences in the number and severity of impacts between the two populations may be an artifact of different data collection methods or may reflect relatively subtle socio-cultural differences in subjective oral health between these populations.

\section{IN BRIEF}

- In a random population sample of adults, one in six people reported impacts 'fairly often' or 'very often' during the preceding year.

- There was striking similarity in the prevalence of problems in everyday life attributed to oral disorders in the UK and Australia.

- Differences were observed in individual impacts, with Australians more likely to report four items categorised as pain and physical disability.

- Dentate Australians tended to report a larger number of impacts occurring at low frequency than dentate people in the UK.

- Population differences in the impact of oral disorders may be an artifact of different collection methods or may reflect subtle sociocultural differences in subjective oral health.

\section{COMMENT}

More attention is being given to the effects of the mouth on everyday life. This field of research is pertinent to dentistry where our aim is to improve the quality of our patients' lives. This focus on the outcome, rather than the process of care, is compatible with broader concepts of health and may allow a better orientation of resources to promote health. Information on health-related quality of life can be used for political purposes (for example, to obtain resources for oral health programmes), for theoretical purposes (to explore determinants of health) and for practical purposes to plan and evaluate oral care.'

This paper reports on a study comparing oral health related quality of life in Australian and UK Adults. The measure used; OHIP14 assesses the degree to which the mouth impacts on normal role function. ${ }^{2,3}$. Dentate participants in Australia were slightly more likely to report having an impact fairly often or very often than in the UK. In contrast people in the UK were significantly more likely to say that they never experienced impacts from their mouth, particularly in relation to pain and physical disability.

The most striking differences were those within each country. The implication being that an average Australian is rather like an average British person, but those averages mask large differences in each population. These findings support a role for both dental and socio-economic status impacting on quality of life and may have policy implications. The data are consistent with the view that in order to reduce inequalities in oral health then one solution is via oral health promotion, including the reduction of social inequalities.

From a theoretical perspective, this study illuminates the area of health-related quality of life and provides insights into the way individuals and populations perceive their oral health. In part the differences between the two countries may be attributed to the use of face-to-face interviews in the UK. Research participants may be less inclined to 'grumble' about trivial impacts face-to-face than they would in a postal questionnaire. But some of the discrepancies may be explained by cultural differences between the two countries. For example, whilst the mouth will affect similar aspects of life in Australia and the UK, it may be that we place greater emphasis on different aspects in the two places. Alternatively, as the authors suggest, there may be differences in expectations about oral health in Australia and the UK. ${ }^{4}$

1. Locker D. Applications of self-reported assessments of oral health outcomes. J Dent Educat 1996; 60: 494-500.

2. Locker D. Measuring oral health: a conceptual framework. Community Dental Health 1988; 5: 3-18.

3. Slade G D. Derivation and validation of a short-form oral health impact profile. Community Dent Oral Epidemiol 1997; 25: 284-290.

4. Gregory J, Gibson B J, Robinson P G. Variation and change in the meaning of ora health related quality of life: a 'grounded' systems approach. Soc Sci Med In press.

Peter G. Robinson

Professor of Dental Public Health, School of Clinical Dentistry, Sheffield

doi:10.1038/sj.bdj.4812261 